\title{
Prevalência de depressão em idosos atendidos em ambulatório de geriatria da região nordeste do Brasil (São Luís-MA)
}

Prevalence of depression in elderly assisted in a geriatrics ambulatory in northeastern Brazil

(São Luis city, state of Maranhão)

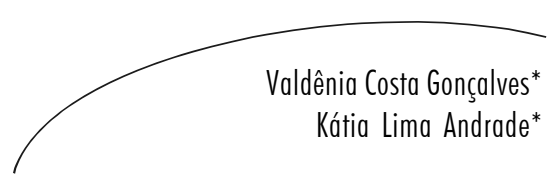

Resumo

Introdução: $\mathrm{O}$ envelhecimento da população é hoje um fenômeno universal e a depressão é um problema de saúde frequente entre os idosos. Objetivos: Determinar a prevalência de depressão em idosos atendidos em ambulatório de geriatria e estabelecer os fatores associados. Metodologia: Foi realizado estudo transversal com aplicação de questionário contendo: variáveis clínicas e epidemiológicas, Escala de Depressão Geriátrica de Yesavage (EDG), WHOQOL-Old e WHOQOL-Abreviado em idosos atendidos no Setor de Geriatria do Programa de Ação Integrada para o Aposentado, de janeiro a abril de 2009, em São Luis-MA. Os dados foram analisados utilizando-se o programa SPSS for Windons 14.0, com aplicação dos testes nãoparamétricos adequados, inclusive correlação de Spearman, considerando as diferenças significantes quando $\mathrm{P}<0,05$. Resultados: Foram avaliados 102 idosos com a idade variando de 60 a 91 anos e a média de $71,5 \pm 6,7$ anos. A maioria eram mulheres $(71,6 \%)$, casados $(64,8 \%)$ e aposentados $(87,3 \%) ; 38,2 \%$ eram hipertensos; $11,8 \%$, diabéticos; $20,6 \%$, com hipertensão e diabetes; $40,2 \%$ procuraram atendimento por doença do sistema osteomuscular; $34,3 \%$, do aparelho circulatório e $14,7 \%$, por doenças endócrinas. A EGD variou de 0 a 13 , com a média de 6,0 $\pm 3,9$. A prevalência de depressão foi de $50 \%$, sendo grave em 13,7\%. Observou-se associação significante entre depressão e idade, sexo, raça e atividade física, refletindo na qualidade de vida dos idosos. Conclusão: A prevalência de depressão foi alta, afetando homens, negros e sedentários, com agravamento conforme avanço da idade e influenciando na piora da qualidade de vida.

\section{Abstract}

Introduction: The aging population is now-a-days a universal phenomenon and depression is a frequent health problem among the elderly. Objectives: To determine the prevalence of depression in elderly outpatients of a Geriatric ambulatory and establish associated factors. Methodology: We conducted a cross-sectional study through a questionnaire containing: clinical and epidemiological variables, Geriatric Depression Scale of Yesavage (GDS), WHOQOL-old and WHOQOL-bref in elderly

*entro Universitário do Maranhão-UNICEUMA. Curso de Medicina. Unidade Renascença. São Luis, MA, Brasil.

Palavras-chave:

Depressão.

Envelhecimento. Idoso.

Pacientes Ambulatoriais.

Escala de Depressão

Geriátrica. 
participating of the Geriatrics Program of Action for Retirement January to April 2009, São Luis-MA. Data were analyzed using SPSS 14.0 for Windows with application of appropriate non-parametric tests, including Spearman correlation, considering differences significant when $\mathrm{P}<0,05$. Results: We studied 102 elderly patients with age ranging from 60 to 91 years and the mean of $71.5 \pm 6.7$ years. Most were women $(71.6 \%)$, married $(64.8 \%)$ and retirees $(87.3 \%) ; 38.2 \%$ were hypertensive; $11.8 \%$, diabetics; $20.6 \%$ with hypertension and diabetes; $40.2 \%$ sought treatment for disease of the musculoskeletal system; $34.3 \%$ of the circulatory system and $14.7 \%$ for endocrine diseases. The EGD ranged from 0 to 13 with an average of $6.0 \pm 3.9$. The prevalence of depression was $50 \%$, considered severe in $13.7 \%$. There was a significant association of depression with age, sex, race and physical activity reflecting in the quality of life of the elderly. Conclusion: The prevalence of depression was high, affecting men, black and sedentary elderly, worsening as they grow older and worsening the quality of life.
Key words: Depression. Aging. Aged. Outpatients. Geriatric Depression Scale.
INTRODUÇÃOO

A doença psiquiátrica mais comum entre os idosos é a depressão ${ }^{1}$ e está associada ao maior risco de morbidade e de mortalidade, ao aumento na utilização dos serviços de saúde, à negligência no autocuidado, à adesão reduzida aos regimes terapêuticos e maiores risco de suicídio. ${ }^{2,3}$

O surgimento da depressão está relacionado a vários fatores biológicos e psicossociais, tais como histórico depressivo ao longo da vida, perda de suporte social e familiar, doenças físicas ou incapacitantes, além do uso de alguns medicamentos que podem desencadear perturbações do humor, como alguns antihipertensivos, corticóides, hipnóticos e outros.,

O idoso deprimido verbaliza de modo diferente suas queixas. ${ }^{5}$ Ele procura um clínico geral, quando muitas vezes os sintomas físicos são um modo de manifestar um sofrimento psíquico em decorrência da depressão. Tristeza, perda de interesse ou prazer, distúrbio do sono, anorexia e perda de peso são alguns dos sintomas apresentados por idosos deprimidos. ${ }^{6}$

Existem várias escalas para avaliar sintomas depressivos, muitas das quais têm sido utilizadas para o rastreamento desses sintomas na população geral, mas a Escala de Depressão Geriátrica (EDG) é um dos instrumentos mais frequentemente utilizados para o rastreamento de depressão em idosos, com perguntas que evitam a esfera das queixas somáticas, ${ }^{7}$ sendo um instrumento já validado no Brasil. ${ }^{8}$

Dentre as vantagens da escala, destacam-se: é composta por perguntas fáceis de serem entendidas; tem pequena variação nas possibilidades de respostas; pode ser autoaplicada ou aplicada por um entrevistador treinado. ${ }^{7}$

A depressão no idoso com frequênciaé secundária a outras doenças, como câncer, Doença de Parkinson, demência, diabetes ou ao uso de medicamentos, como anti-hipertensivos, corticosteróides, entre outros. Juntem-se a isto as perdas: fim da vida profissional, da reprodutiva, diminuição da renda, do poder, morte dos amigos, dos familiares, do cônjuge. ${ }^{3,9} \mathrm{E}$ dentro dessa realidade é necessário o conhecimento sobre depressão em idosos para distinguir as alterações normais das mórbidas visando ao diagnóstico precoce e correto que possa orientar intervenção adequada, assim como prevenir os fatores de risco associados à depressão e promover melhoria da qualidade de vida dosidosos. $^{2}$

É fundamental que profissionais de saúde aprimorem cada vez mais seus conhecimentos sobre depressão em idosos, pois é o clínico ou geriatra que o idoso procura como demanda específica de sintomas disfóricos, com quadros inespecíficos de queixas como cansaço ou fadiga ou ainda, mesclando sintomas de depressão com quadro clínico de outras patologias. Nesse sentido, o uso sistemático de escalas de depressão e uma correta avaliação podem facilitar a detecção 
precoce da doença e tornar possível uma intervenção mais adequada sobre os indivíduos, que poderão, assim, experimentar essa fase com melhor qualidade de vida. ${ }^{2}$ Portanto, é imprescindível haver estudos específicos com grupos etários de idade avançada, por parte de profissionais da área da saúde, para uma melhor compreensão do envelhecimento e assim possibilitar a criação de alternativas de intervenção visando ao bem-estar das pessoas idosas.

O objetivo do presente trabalho é determinar a prevalência de depressão em idosos atendidos em ambulatório de geriatria da Região Nordeste.

\section{METODOLOGIA}

População, amostra, local e desenho do estudo

Foram avaliados pacientes atendidos no Ambulatório de Geriatria do Programa de Apoio ao Aposentado (PAI) do Governo do Estado do Maranhão, no período de janeiro a abril de 2009. O ambulatório de Geriatria do PAI está localizado no município de São Luís, MA, tendo sido em 1992 com a missão de resgatar o respeito e a dignidade do aposentado da esfera estadual na sua dimensão biopsicossocial, apresentando uma média de 80 atendimentos mensais.

Todos os idosos atendidos foram convidados a participar do estudo e todos concordaram em participar e assinar o Termo de Consentimento Livre e Esclarecido (TCLE). Nenhum idoso foi excluído por incapacidade de responder às perguntas do questionário ou à ausência de um responsável legal, ou não-interesse em participar do estudo e/ou a não-assinatura do TCLE.

Foi realizado estudo do tipo transversal, com aplicação de questionário.

\section{Instrumentos}

\section{Ficha de identificação e de dados sócio-demográficos}

A ficha de identificação e de dados sóciodemográficos incluiu as seguintes variáveis: sexo, idade, naturalidade, grupo racial, estado civil, escolaridade, moradia, tipo de domicílio, domicílio na companhia de quem, ocupação, atividade física realizada, uso de cigarro e bebida (quantidade e frequência). Também avaliou as seguintes questões: diagnóstico principal da doença que levou o idoso a procurar consulta médica, utilizando o Código Internacional de Doenças $10^{\mathrm{a}}$ versão (CID 10 ), e presença de comorbidades.

\section{Escala de Depressão Geriátrica}

A Escala de Depressão Geriátrica (EDG) já está validada para uso no Brasil, ${ }^{8}$ com 15 itens. Foi preconizado o ponto de corte proposto por Freitas et al. ${ }^{10}$ maior que seis, sugestivo de depressão. A EDG busca avaliar a satisfação com a vida, interrupção de atividades, aborrecimento, humor, isolamento, energia, alegria e problemas relacionados à memória.

\section{WHOQOL-Old e WHOQOL-Abreviado}

Questionários de qualidade de vida conforme manual do instrumento validado no Brasil, ${ }^{11,12} \mathrm{com}$ perguntas relacionadas ao funcionamento do sensório, autonomia, atividades passadas, presentes e futuras, participação social, morte e morrer e intimidade e envolvendo ainda os domínios físico, psicológico, relações sociais e meio ambiente.

\section{Procedimentos para coleta de dados}

Todos os idosos foram informados do estudo e preencheram o TCLE. O questionário foi aplicado, sob forma de entrevista, pelo pesquisador ao idoso de maneira clara e concisa, sem que ocorresse interferência do pesquisador na resposta. Em caso de dúvidas no entendimento, o entrevistador repetia a questão até que o indivíduo da pesquisa escolhesse a alternativa que julgasse correta.

O projeto foi aprovado pelo Comitê de Ética em Pesquisa - CEP do Centro Universitário do 
Maranhão - UNICEUMA, São Luís (MA), Brasil (protocolo n ${ }^{\circ}$ 00964/08).

Procedimentos para análise dos dados

Os dados coletados foram processados no programa de Epi Info 2004, fornecido pelo Centers for Disease Control and Prevention (CDC) e foram analisados utilizando-se o pacote estatístico SPSS (Statistical Package for Social Sciences), versão 14.0. A análise descritiva resumiu as variáveis em percentagens, frequências e medidas de tendência central e de dispersão. Foram aplicados testes pertinentes de significância estatística em análise univariada.

No estudo de correlação entre as variáveis numéricas, foi utilizado o coeficiente de correlação de Spearman (rho), considerando-se a amostragem não-paramétrica. Foi adotado intervalo de confiança de $95 \%$, com nível de significância de $\mathrm{p}<0,05$.

\section{RESULTADOS}

Foram entrevistados 102 idosos na faixa etária entre 60 e 91, com a média de 71,5 $\pm 6,7$ e predomínio do sexo feminino (71,6\%), caucasianos (62,7\%), aposentados (87,3\%), casados (64,8\%) e idosos que não consomem bebidas alcoólicas $(87,3 \%)$. A tabela 1 mostra as características sóciodemográficas da amostra em estudo. Quanto à situação de moradia, prevaleceu a condição de moradia própria (94,1\%); a escolaridade mais prevalente foi o ensino médio $(61,8 \%)$. Dos entrevistados, $40,2 \%$ procuraram o ambulatório com queixas de doenças do sistema osteomuscular e do tecido conjuntivo.

Tabela 1 - Características clínico-demográficas dos 102 pacientes atendidos, no período de janeiro a março de 2009, no ambulatório de Geriatria do Programa de Apoio ao Aposentado (PAI) do Governo do Estado do Maranhão. São Luis, MA, 2009.

\begin{tabular}{|c|c|c|c|}
\hline & Características & $\mathrm{n}$ & $\%$ \\
\hline \multirow[t]{3}{*}{ Idade } & Idoso jovem (60 a 69 anos) & 44 & 43,1 \\
\hline & Meio-idoso (70 a 79 anos) & 46 & 45,1 \\
\hline & Idoso Velho ( 80 ou mais anos) & 12 & 11,8 \\
\hline \multirow[t]{2}{*}{ Sexo } & Masculino & 29 & 28,4 \\
\hline & Feminino & 73 & 71,6 \\
\hline \multirow[t]{3}{*}{ Raça } & Branca & 64 & 62,7 \\
\hline & Mulato & 22 & 21,6 \\
\hline & Negro & 16 & 15,7 \\
\hline \multirow[t]{4}{*}{ Estado Civil } & Solteiro & 10 & 9,8 \\
\hline & Casado & 66 & 64,8 \\
\hline & Divorciado & 3 & 2,9 \\
\hline & Viúvo & 23 & 22,5 \\
\hline \multirow[t]{4}{*}{ Naturalidade } & São Luís & 56 & 54,9 \\
\hline & Outro Município do Estado & 44 & 43,1 \\
\hline & Outro Estado & 1 & 1,0 \\
\hline & Outro País & 1 & 1,0 \\
\hline
\end{tabular}


Tabela 1 - Características clínico-demográficas dos 102 pacientes atendidos, no período de janeiro a março de 2009, no ambulatório de Geriatria do Programa de Apoio ao Aposentado (PAI) do Governo do Estado do Maranhão. São Luis, MA, 2009. (continuação)

\begin{tabular}{llll}
\hline & Características & $\mathrm{n}$ & $\%$ \\
\hline \multirow{2}{*}{ Escolaridade } & Ensino Fundamental & 16 & 15,7 \\
& Ensino Médio & 63 & 61,8 \\
& Ensino Superior & 22 & 21,6 \\
& Ignorado & 1 & 0,9 \\
& & & \\
Diagnóstico principal & Doenças endócrinas. nutricionais e metabólicas & 15 & 14,7 \\
& Transtornos mentais e comportamentais & 2 & 2,0 \\
& Doenças do sistema nervoso & 1 & 1,0 \\
& Doenças do aparelho circulatório & 35 & 34,3 \\
& Doenças do sistema osteomuscular e do tecido conjuntivo & 41 & 40,2 \\
& Quedas & 8 & 7,8 \\
& & & \\
Presença de co-morbidades & Hipertensão & 39 & 38,2 \\
& Diabetes & 12 & 11,8 \\
& Hipertensão e Diabetes & 21 & 20,6 \\
& Nenhuma & 30 & 29,4 \\
Tabagismo & & & \\
& Nunca fumou & 87 & 85,3 \\
& Ex-fumante & 14 & 13,7 \\
Atividade física & Fumante & 1 & 1,0 \\
& Sedentário & & \\
& Irregular & 24 & 23,5 \\
& Regular & 32 & 31,4 \\
& & 46 & 45,1 \\
\hline
\end{tabular}

A pontuação da EDG variou de 0 a 13, com média de 6,0 $\pm 3,9$. Observou-se que $50 \%$ dos idosos apresentaram pontuações sugestivas de depressão, alcançando mais de seis pontos no escore utilizado. Entre os deprimidos, $13,7 \%$ foram caracterizados como sugestivos de depressão grave (maior que 10 pontos na EDG). 
A tabela 2 apresenta os fatores associados à depressão nos idosos, com significância para sexo masculino, negros e sedentários. Não foram observadas diferenças significantes relacionadas com co-morbidades e estado civil dos idosos, mas no quesito atividade física, parcela expressiva da amostra $(87 \%)$ praticava atividades regulares, não apresentando sintomas de depressão.

Tabela 2 - Fatores associados à depressão em 102 pacientes atendidos no ambulatório de Geriatria do PAI do Governo do Estado do Maranhão de janeiro a abril de 2009. São Luis, MA, 2009.

\begin{tabular}{lllll}
\hline \multirow{2}{*}{ Fatores Associados } & \multicolumn{4}{c}{ Depressão n (\%) } \\
\cline { 2 - 5 } & Não deprimido & Deprimido & Total & $\mathrm{P}$ \\
\hline IDADE & & & & $0,001 *$ \\
Idoso jovem & $26(59,1)$ & $18(40,9)$ & $44(43,1)$ & \\
Meio-idoso & $23(50)$ & $23(50)$ & $46(45,1)$ & \\
Idoso velho & $2(16,7)$ & $10(83,3)$ & $12(11,8)$ & \\
SEXO & $6(20,7)$ & $23(79,3)$ & $29(28,4)$ & $0,000 *$ \\
Masculino & $45(61,6)$ & $28(38,4)$ & $73(71,6)$ & \\
Feminino & & & & $0,000 *$ \\
RAÇA & $39(60,9)$ & $25(39,1)$ & $64(62,7)$ & \\
Branco & $11(50)$ & $11(50)$ & $22(21,6)$ & \\
Mulato & $1(6,2)$ & $15(93,8)$ & $16(15,7)$ & $0,000 *$ \\
Negro & & & & \\
ATIVIDADE FÍSICA & $5(20,8)$ & $19(79,2)$ & $24(23,5)$ & \\
Sedentário & $6(18,8)$ & $26(81,3)$ & $32(31,4)$ & \\
Irregular & $40(87)$ & $6(13)$ & $46(45,1)$ & \\
Regular & &
\end{tabular}

* Teste do qui-quadrado; **Teste Exato de Fisher.

As médias de escores da idade, EDG e dos domínios do WHOQOL-Old e do WHOQOLAbreviado tiveram distribuição de acordo com a tabela 3. No que se refere aos domínios do
WHOQOL-Old e WHOQOL-Abreviado em correlação aos escores da EDG, a pesquisa aponta resultados estatisticamente significativos com $p$ $<0,01$. 
Tabela 3 - Variáveis quantitativas avaliadas em 102 pacientes atendidos no ambulatório de Geriatria do PAI do Governo do Estado do Maranhão de janeiro a abril de 2009. São Luis, MA, 2009.

\begin{tabular}{llllll}
\hline & Variáveis numéricas & Média & $\begin{array}{l}\text { Desvio } \\
\text { Padrão }\end{array}$ & Mínimo & Máximo \\
\hline Idade & & 71,5 & 6,7 & 60,0 & 91,0 \\
EGD & & 6,0 & 3,9 & 0,0 & 13,0 \\
WHOQOL OLD & Sensório & 12,9 & 2,5 & 8,0 & 18,0 \\
& Autonomia & 12,6 & 2,4 & 7,0 & 17,0 \\
& Atividades & 13,1 & 2,0 & 9,0 & 16,0 \\
& Social & 12,7 & 2,4 & 8,0 & 16,0 \\
& Morte & 12,3 & 2,9 & 8,0 & 20,0 \\
& Intimidade & 12,7 & 2,5 & 8,0 & 16,0 \\
WHOQOL & Escore total & 76,5 & 12,7 & 53 & 103,0 \\
ABREVIADO & Físico & 55,5 & 12,5 & 28,6 & 82,1 \\
& Psicológico & 58,3 & 11,7 & 33,3 & 79,1 \\
& Social & 47,6 & 15,9 & 16,6 & 75,0 \\
& Ambiental & 53,4 & 11,1 & 25,0 & 75,0 \\
\hline
\end{tabular}

$\mathrm{Na}$ tabela 4, ao se analisar as médias de pontuações no WHOQOL-Old e WHOQOLAbreviado entre os idosos com e sem depressão, foram encontrados maiores índices de depressão nos domínios morte $(10,6 \pm 2,1)$ e no social $(37,9$ $\pm 11,5)$, respectivamente.

Tabela 4 - Distribuição das variáveis qualitativas associadas à depressão em 102 pacientes atendidos no ambulatório de Geriatria do PAI do Governo do Estado do Maranhão de janeiro a abril de 2009. São Luis, MA, 2009.

\begin{tabular}{llcl}
\hline & \multicolumn{3}{c}{ Depressão } \\
Variáveis & Não Deprimido & Deprimido & rho(*) \\
\hline Idade & $69,9 \pm 5,4$ & $73,2 \pm 7,4$ &, $307(* *)$ \\
WHOQOL OLD & & & \\
Sensório & $14,5 \pm 1,8$ & $11,3 \pm 2,1$ &,$- 719(* * *)$ \\
Autonomia & $14,3 \pm 1,8$ & $11,0 \pm 1,7$ &,$- 734(* *)$ \\
Atividades & $14,7 \pm 1,4$ & $11,6 \pm 1,2$ &,$- 809(* * *)$ \\
Social & $14,3 \pm 1,8$ & $11,0 \pm 1,8$ &,$- 768(* *)$ \\
Morte & $14,0 \pm 2,7$ & $10,6 \pm 2,1$ &,$- 605(* * *)$ \\
Intimidade & $14,4 \pm 1,7$ & $11,0 \pm 1,9$ &,$- 730(* * *)$ \\
Escore Total & $86,5 \pm 8,6$ & $66,6 \pm 7,3$ &,$- 854(* * *)$ \\
WHOQOL ABREVIADA & & & \\
Físico & $63,8 \pm 8,2$ & $47,1 \pm 10,4$ &,$- 745(* * *)$ \\
Psicológico & $67,4 \pm 8,1$ & $49,2 \pm 6,5$ &,$- 863(* * *)$ \\
Social & $57,3 \pm 13,6$ & $37,9 \pm 11,5$ &,$- 735(* * *)$ \\
Ambiental & $60,4 \pm 10,0$ & $46,5 \pm 7,1$ &,$- 642(* 8)$ \\
\hline
\end{tabular}

* *Coeficiente de correlação de Spearman . **r $<0,01$. 
Foi observada associação significante entre depressão e qualidade de vida, em que as variáveis mostraram que quanto maior é a ocorrência de sintomas sugestivos de depressão, menor é a pontuação para qualidade de vida.

\section{DISCUSSÃO}

Neste estudo, os dados sócio-demográficos obtidos estão de acordo com a literatura nos aspectos: sexo (feminino), raça (branca), estado civil (casado), ocupação (aposentado) e comorbidade (hipertensão), como os mais predominantes na população idosa. ${ }^{13}$

A depressão é uma condição que afeta muitas pessoas em alguma fase de suas vidas, seja como humor transitório, ao se sentir abatido ou melancólico, ou como uma forma mais séria, que pode prejudicar o desempenho físico e psicológico. ${ }^{1,2,9}$

Diferentes estudos têm sugerido que o envelhecimento apresenta estreita relação com a presença de depressão. Para Mazzeo et al., ${ }^{14}$ a depressão é uma das mais frequentes desordens de saúde mental registradas com o avanço da idade.

A prevalência de sintomas depressivos na presente amostra de idosos foi muito alta. Camarano ${ }^{13}$ relata que a ocorrência de depressão está associada a fatores como idade, sexo, estado civil, condições sociais e atividades físicas. $\mathrm{O}$ presente estudo mostrou que as variáveis idade, sexo, raça e atividade física tiveram relevância. Observou-se que quanto maior a idade do idoso, maior a sintomatologia depressiva. Essa associação entre depressão e idade parece estar relacionada especialmente à perda da capacidade funcional e ao declínio da saúde com o passar do tempo..$^{15,16}$

A análise indicou escores de sintomas depressivos estatisticamente maiores nos homens, o que não é corroborado por outros estudos que mostram maior frequência de mulheres em relação aos homens. ${ }^{17,18} \mathrm{O}$ que a literatura relata é a ideia de que a depressão é mais diagnosticada em mulheres porque elas procuram mais os serviços de saúde, ${ }^{18,19}$ e também a hipótese sobre a longevidade da mulher, que estaria ligada a fatores biológicos, padrões de comportamento e estilo de vida mais saudáveis. ${ }^{13}$

No estudo, também se observou associação de depressão em idosos solteiros e viúvos e negros, o que não é encontrado em nossa literatura.

É importante ressaltar que as características da população estudada não se assemelham às de outros estudos com idosos no Brasil que mostram maior frequência de mulheres idosas, com baixa escolaridade. Entretanto, nossa amostra foi composta, em sua maioria, por idosas com nível de escolaridade médio a elevado. Estudos relatam que a escolaridade exerceria um papel protetor para sintomas depressivos, relação também encontrada neste estudo. Outros estudos também têm demonstrado associação entre menor escolaridade e maior número de idosos deprimidos. ${ }^{2,20,21}$

Todavia, é importante destacar as características da amostra estudada, que foi composta, em sua maioria, por mulheres de classe média, aposentadas, com nível de escolaridade médio a elevado. Este fato se deve à participação pouco expressiva dos homens idosos em programas para a terceira idade. ${ }^{22}$

Dentre os fatores associados, as comorbidades podem ser responsáveis por uma pequena parte dos sintomas somáticos, que podem ser vistos como sintomas da depressão e não como expressão de saúde física precária. ${ }^{23}$ Esse conjunto de fatores é identificado para o desenvolvimento de sintomatologia depressiva. As mudanças nos papéis sociais, tais como a aposentadoria, frequentemente ocasionam a diminuição $d a$ autoestima, do ritmo das atividades e do interesse pelas atividades cotidianas..$^{20}$

Segundo Papalia et al., ${ }^{24}$ uma forte rede de amigos e de familiares pode ajudar os idosos a evitar ou enfrentar a depressão. Os sentimentos gerados nos idosos pelo processo de envelhecimento são vividos, na maioria das vezes, como algo novo e desestabilizador, resultando em 
ansiedade, medo, conflito, insegurança e, principalmente, sentimentos de solidão. Dessa forma, determinados contextos afetivos podem tanto desencadear quanto proteger os indivíduos contra o surgimento de sintomatologia depressiva. O risco de depressão é aumentado na ausência de relacionamentos afetivos, de amizades e de vínculos de confiança, com cônjuge, parceiro ou amigo. ${ }^{25}$

Uma associação muito importante é com atividade física, que tem como objetivo principal a valorização do indivíduo idoso, enquanto ser pensante ativo e participante da sociedade ${ }^{26}$. Resultados de estudos feitos por Gobbi et al. ${ }^{27}$ com idosos praticantes de atividade física regular mostraram efeitos positivos do exercício físico com relação à depressão. Para Stoppe Junior \& Louzã Neto, ${ }^{28}$ os índices de depressão são menores em idosos que praticam atividade física regular, o que possibilita melhoria no aspecto emocional, aumento da autoestima, humor, bem-estar, diminuição do estresse, da ansiedade e da tensão.

Uma associação entre menor intensidade de sintomas depressivos e uma melhor qualidade de vida na terceira idade foi encontrada em nosso estudo, alertando para o fato de que depressão influencia negativamente sobre a qualidade de vida.

\section{CONCLUSÃO}

Atualmente, a idade avançada é descrita como desprovida de força, incapaz de prazer, solitária e repleta de amargura. No passado, certas sociedades garantiam ao idoso o poder, a honra e o respeito. Entretanto, na sociedade moderna, consumista e imediatista, os idosos são encarados como um peso social, sempre recebendo benefício e nada oferecendo em troca. Os valores da juventude predominam como os de beleza, de energia e de ativismo. ${ }^{29}$ Assim, conclui-se que às limitações naturais físicas são acrescidas aquelas colocadas pela sociedade, fruto de preconceitos e estereótipos sociais. Tais fatos podem explicar, em parte, a alta prevalência de depressão encontrada na presente pesquisa.

Há necessidade de se criar programas nacionais para os idosos, com o fim de promover participações em movimentos sociais e envolvimento com atividades culturais, desportivas e de lazer com incentivo à prática de atividade física regular. Esses programas devem ser compatíveis com a disponibilidade e o interesse da população idosa envolvida, levando em consideração suas possibilidades e seus limites pessoais, levando à diminuição da sintomatologia depressiva neste grupo etário.

\section{AGRADECIMENTOS}

Agradecimento a todos que participaram e contribuíram com a pesquisa, idosos e Dra. Claudia Rego, médica do ambulatório de Geriatria do PAI. 


\section{REFERENNCIAS}

1. Blazer DG. Depressão. In: Abrams WB, Berkow R. Manual Merck de geriatria. São Paulo: Roca; 1994. p. 1086-90.

2. Veras RP. Atenção preventiva ao idoso: uma abordagem de saúde coletiva. In: Papaleo Netto, organizador. Gerontologia: a velhice e o envelhecimento em visão globalizada. São Paulo: Atheneu; 2002. p. 383-93.

3. Toledo MAV, Santos Neto LS. Depressão no idoso. In: Hargreaves LH, organizador. Geriatria. Brasília: Prodasen; 2006. p. 545-52.

4. Gordilho A. Depressão, ansiedade outros distúrbios afetivos e suicídio. In: Freitas EV, et al. Tratado de geriatria e gerontologia. Rio de Janeiro: Guanabara Koogan; 2002. p. 204-15.

5. Forlenza OV, Caramelli P. Neuropsiquiatria geriátrica. São Paulo: Atheneu; 2000.687 p.

6. Carvalho Filho ET, Papaléo Netto M. Geriatria: fundamentos, clínica e terapêutica. São Paulo: Atheneu; 2004.

7. Paradela EMP, Lourenço RA, Veras RP. Validação da escala de depressão geriátrica em um ambulatório geral. Rev. Saúde Pública. [periódico online]. $2005 \mathrm{dez}$; 39(6): 918-23. Disponível em: http://www.scielosp.org/ scielo.php?script $=$ sci_arttext\&pid = S003489102005000600008.

8. Almeida OP, Almeida SA. Confiabilidade da versão brasileira da escala de depressão em geriatria (GDS) versão reduzida. Arq Neuropsiquiatr 1999; 57(2B): 421-6.

9. Cohen GD. Alterações e padrões normais da doença psiquiátrica. In: Abrams WB, Robert B. Manual Merck de geriatria. São Paulo: Roca; 1994. p. 1065-75.

10. Freitas EV, et al. Tratado de geriatria e gerontologia. Rio de janeiro: Guanabara Koogan; 2006.

11. Fleck MPA, Chachamovich E, Trentini CM. Projeto WHOQOL-OLD: método e resultados de grupos focais no Brasil. Rev. Saúde Pública 2003 dez; 37(6): 793-9.

12. Fleck MPA, Louzada S, Xavier M, et al. Aplicação da versão em português do instrumento abreviado de avaliação de qualidade de vida "WHOQOL-bref". Rev. Saúde Pública 2000; 34(2):178-83.

13. Camarano AA. Envelhecimento da população brasileira: uma contribuição demográfica. Rio de Janeiro: IPEA; 2002. (Texto para discussão; 858).
14. Mazzeo RS, et al. Exercício e atividade física para pessoas idosas. Revista Brasileira Atividade Física \& Saúde 1998 jun; 3(1): 48-78.

15. Trentini CM, et al. A influência dos sintomas somáticos no desempenho dos idosos no Inventário de Depressão de Beck (BDI). Revista Brasileira Psiquiátrica 2005; 27(2): 119-23.

16. Carvalho VFC, Fernandez MED. Depressão no Idoso. In: Papaléo Netto M, organizador. Gerontologia. São Paulo: Atheneu; 2002. p. 160-73.

17. Stoppe AJ. Aspectos clínicos da depressão em idosos. Revista Psiquiatria Clínica 1994; 21(4): 121-8.

18. Stella F, et al. Depressão no idoso: diagnóstico, tratamento e benefícios da atividade física. Motriz, Rio Claro 2002 ago./dez; 8(3): 91-8.

19. Oliveira DAAP, Gomes L, Oliveira RF. Prevalência de depressão em idosos que frequentam centros de convivência. Rev. Saúde Pública 2006 ago; 40(4): 734-6.

20. Forlenza OV. Fatores de risco associados à morbidade psiquiátrica em idosos. In: Forlenza OV, Almeida OP, editores. Depressão e demência no idoso: tratamento psicológico e farmacológico. São Paulo: Lemos; 1997. p. 25-46.

21. Oliveira MR. Depressão na velhice: aspectos epidemiológicos. [dissertação] Salvador: Escola de Enfermagem da Universidade Federal da Bahia; 1992.

22. Silveira LD, Duarte MFS. Níveis de depressão, hábitos e aderência a programas de atividades físicas de pessoas diagnosticadas com transtorno depressivo. Revista Brasileira de Cineantropometria \& Desempenho Humano Florianópolis 2004 nov; 6(2): 36-44.

23. Zimerman GI. Velhice: Aspectos Biopsicossociais. Porto Alegre: Artmed; 2000.232 p.

24. Papalia DE, Olds SW, Feldman RD. Desenvolvimento humano. Porto Alegre: Artmed; 2006.

25. Marchand EAA. A Influência da atividade física sobre a saúde mental de idosos. Revista Virtual EF Artigos 2003 jun; 1(4).

26. Mazo GZ, et al. Tendência a estados depressivos em idosos praticantes de atividade física. Revista Brasileira Cineantropometria \& Desempenho Humano 2005; 7(1): 45-9.

27. Gobbi S, et al. Atividade física e saúde mental no idoso. In: Encontro Paulista de Neuropsiquiatria \& Saúde Mental do Idoso 
2003, São Paulo. São Paulo: Sociedade Paulista de Psiquiatria Clínica, 2003.

28. Stoppe Júnior A, Louzã Neto MR. Depressão na terceira idade: apresentação clínica e abordagem terapêutica. São Paulo: Lemos; 1999. p. 206.
29. Papaléo Netto M. O estudo da velhice no século $\mathrm{XX}$ : histórico, definição do campo e termos básicos. In: Freitas EV, et al. Tratado de geriatria e gerontologia. Rio de Janeiro: Guanabara Koogan; 2002. p. 2-12.

Recebido: 23/10/2009

Aprovado: 30/3/2010 
